\title{
The electroneutral sodium/bicarbonate cotransporter containing an amino terminal 123-amino-acid cassette is expressed predominantly in the heart
}

\author{
Deborah S. Cooper ${ }^{1}$, Hye Jeong Lee ${ }^{2}$, Han Soo Yang ${ }^{1}$, Joseph Kippen ${ }^{1}$, C. Chris Yun ${ }^{2}$ \& \\ Inyeong Choi ${ }^{1, *}$ \\ ${ }^{1}$ Department of Physiology, Emory University School of Medicine, 615 Michael Street, Atlanta, GA 30322 \\ USA; ${ }^{2}$ Division of Digestive Disease, Department of Medicine, Emory University School of Medicine, \\ Atlanta, GA 30322 USA
}

Received 7 December 2005; accepted 5 February 2006

(C) 2006 National Science Council, Taipei

Key words: acid-base regulation, bicarbonate transporter, sodium bicarbonate symporter

\begin{abstract}
Summary
In this study, we examined the tissue-specific expression of two electroneutral $\mathrm{Na} / \mathrm{HCO}_{3}$ cotransporter $(\mathrm{NBCn} 1)$ variants that differ from each other by the presence of the N-terminal 123 amino acids (cassette II). A rat Northern blot with the probe to nucleotides encoding cassette II detected a $9 \mathrm{~kb}$ NBCn1 mRNA strongly in the heart and weakly in skeletal muscles, but absent from most of the tissues including kidney, brain, and pancreas. In the rat heart, PCR with primers flanking cassette II preferentially amplified a DNA fragment that lacked cassette II. However, in the human heart, PCR preferentially amplified a fragment that contained cassette II. This larger PCR product was found virtually in all regions of the human cardiovascular system with strong amplification in the apex, atrium, and atrioventricular nodes. These findings indicate that the variant containing cassette II is almost absent in tissues including brain, kidney, and pancreas, where NBCn1 has been extensively examined.
\end{abstract}

The proton concentration in the cardiovascular system is important for maintaining cardiac function (for review see Ref [1]). Intracellular acidification decreases contractility by affecting almost all steps in excitation-contraction coupling. Cardiac cells have precise regulatory mechanisms to extrude acids from the cytoplasm. More than $40 \%$ of acid extrusion in cardiac myocytes occur by $\mathrm{Na} /$ $\mathrm{HCO}_{3}$ transport [2]. The carrier protein is proposed as an $\mathrm{Na} / \mathrm{HCO}_{3}$ cotransporter with the 1:1 stoichiometry of $\mathrm{Na}^{+}$versus $\mathrm{HCO}_{3}^{-}$[2]. The electroneutral $\mathrm{Na} / \mathrm{HCO}_{3}$ cotransporter (NBCn1) was cloned from the heart and vascular smooth muscle 6-7 years ago [3, 4]. Many studies have subsequently characterized its cellular and physiological

*To whom correspondence should be addressed. Fax: + 1-404727-2648; E-mail: ichoi@emory.edu function in epithelial and non-epithelial tissues (for review see Ref. [5]). While these provide useful information on physiological properties of $\mathrm{NBCn} 1$, recent reports suggest that the transporters in many tissues appear different from the initially identified cardiovascular clone; $\mathrm{NBCn} 1$ in adult brain and kidney lacks, in the $\mathrm{N}$-terminal domain, a region of 123 amino acids (cassette II) that comprise $\sim 10 \%$ of the total amino acids in the protein [6,7]. Identification of this deletion variant led us to reinvestigate the cardiac tissue specificity of NBCn1.

For experimental methods, we used Northern blot and PCR to distinguish the deletion variant from the non-deletion variant in rat and human tissues. A multiple-tissue Northern blot membrane of adult rats was purchased from BD Bioscience 
(Palo Alto, CA). The nucleotides (964-1,334 of rat NBCn1; GenBank accession number NM_058211) corresponding to cassette II were ${ }^{32} \mathrm{P}$-labeled and hybridized with the membrane at $68^{\circ} \mathrm{C}$ for $2 \mathrm{~h}$ (probe concentration: $0.6 \times 10^{6} \mathrm{cpm} / \mathrm{ml}$ ). The membrane was washed in $2 \times \mathrm{SSC} / 0.1 \% \mathrm{SDS}$ for $40 \mathrm{~min}$, and then in $0.1 \times \mathrm{SSC} / 0.1 \% \mathrm{SDS}$ for $1 \mathrm{~h}$ at $55^{\circ} \mathrm{C}$. The autoradiograph was done $24 \mathrm{~h}$ later. For PCR, total RNA was extracted from adult rat (SpragueDawley) whole heart using Trizol (Life Technologies, Gaithersburg, MD) according to the manufacturer protocol. cDNA was synthesized using Superscript Reverse Transcriptase (Invitrogen, Carlsbad, CA) primed with random hexamers. Primers were designed from the flanking regions of cassette II; 819-846 of rat NBCn1 (forward primer) and 2,2662,295 (reverse primer). In addition, cDNAs of human cardiovascular system (BD Bioscience) were purchased. Primers corresponded to 135-164 (forward) and 1,662-1,691 (reverse) of human NBCn1 sequence (NBC3; GenBank accession number NM_003615). PCR was done in 30 cycles of $94{ }^{\circ} \mathrm{C}$ for $30 \mathrm{~s}, 65{ }^{\circ} \mathrm{C}$ for $45 \mathrm{~s}$, and $72{ }^{\circ} \mathrm{C}$ for $1 \mathrm{~min}$. PCR was repeated minimum three times to verify the results.

Figure 1(A) shows the rat northern blot probed with cassette II. A $9 \mathrm{~kb}$ NBCn1 mRNA was predominantly detected in the heart. The signals in other tissues were very weak or negligible. In particular, the NBCn1 mRNA was almost absent in spleen and testis, kidney, and brain, where a probe to the transmembrane domains of $\mathrm{NBCn} 1$ detected moderate to strong signals [3]. The absence of signals in the brain and kidney is consistent with recent reports that the transporter in these tissues lacks cassette II $[6,7]$. Our data are also similar to the data from human northern blot, except that, in human, NBCn1 signals are strongly detected in both heart and skeletal muscles [4]. Figure 1(B) shows the relative abundance of the NBCn1 variant containing cassette II in the rat heart. PCR with primers flanking cassette II preferably generated an $1.0 \mathrm{~kb}$ product, which corresponds to the deletion variant. The $1.5 \mathrm{~kb}$ product, which corresponds to the non-deletion variant, was very rare. These data indicate that only a small fraction of the total transporters in the rat heart has cassette II. In contrast, when PCR was repeated on the human heart with similar primers, an $1.5 \mathrm{~kb}$ product was preferentially amplified (Figure 1C). This larger PCR product was found virtually in all regions of the human cardiovascular system we examined. The smaller product was also amplified in most tissues though its abundance was minor in the apex of the heart, atrium, and atrioventricular nodes. Therefore, the relative expression of deletion and non-deletion variants varies between rat and human hearts and among different regions of the heart.

The significance of our data is the almost exclusive presence of the cassette II-containing NBCn1 variant in the cardiovascular system.

A
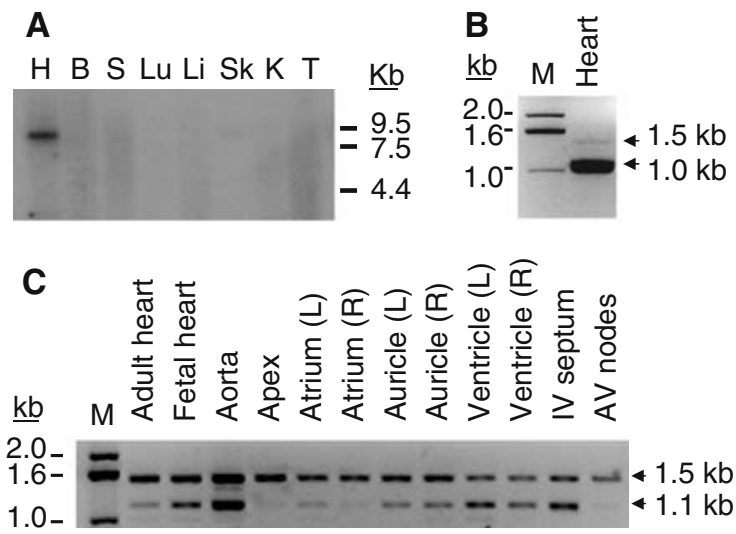

Figure 1. Cardiac expression of NBCn1 variants. (A) Northern blot of rat tissues probed to nucleotides $964-1,334$ (encoding N-terminal cassette II) of NBCn1. H, heart; B, brain; S, spleen; Lu, lung; Li, liver; Sk, skeletal muscle; K, kidney; T, testis. (B) PCR of rat whole heart. Primers were designed from the conserved flanking regions of cassette II. The $1.0 \mathrm{~kb}$ PCR product represents NBCn1 without cassette II, and the $1.5 \mathrm{~kb}$ product represents the one with the cassette. (C) PCR of human cardiovascular system. Primers were designed to amplify a $1.1 \mathrm{~kb}$ product for $\mathrm{NBCn} 1$ without cassette II and a $1.5 \mathrm{~kb}$ product for the one with the cassette. M, molecular marker; IV, interventricular; AV, Atrioventricular. L and R in parenthesis are left and right. 
However, its relative abundance differs between rat and human hearts. Most NBCn1 variants in the rat heart lack cassette II, whereas most variants in the human heart contain the cassette. It is important to note that the transporter initially identified in the cardiovascular systems of rat and human is the non-deletion clone containing cassette II. Our northern blot data show that this clone is almost undetectable in tissues including brain, kidney, and pancreas, where NBCn1 has been extensively examined in vivo and in vitro. Therefore, caution is required when studying NBCn1 in these tissues. Nevertheless, we do not insist that the non-deletion variant is expressed only in the heart (and vascular smooth muscle). It may be possible that the non-deletion variant is also present in other tissues we did not examine in this study.

The function of cassette II is unclear. Cassette II does not directly alter the biophysical properties of the transporter [7], thus raising the possibility of transporter regulation. The cytoplasmic $\mathrm{N}$ - and C-terminal domains of other $\mathrm{Na} / \mathrm{HCO}_{3}$ transporters have been proposed to affect protein expression and trafficking, cellular signaling, and interactions with other proteins $[5,8]$. The non-deletion variant containing cassette II has a slow expression on Xenopus oocytes membranes compared to the one without the cassette (unpublished observation). Thus, the function of cassette II would likely relate to protein trafficking or retention. Alternatively, cassette II might involve interactions with other proteins. We note that the $\mathrm{Cl} / \mathrm{HCO}_{3}$ exchanger AE1 has a binding motif for ankyrin in the region corresponding to cassette II of NBCn1. Thus, it is plausible that cassette II in NBCn1 may be a site for docking other cytosolic proteins.

In conclusion, the presence of two NBCn1 variants in the cardiovascular system is an interesting finding in that their dual expression could be advantageous to some cardiac cells, where $\mathrm{pH}_{\mathrm{i}}$ and ion gradients have to be carefully regulated.
Nonetheless, understanding the mechanism of such regulation will require more complicated investigation.

\section{Acknowledgements}

We are grateful to Eun Jung Shin for PCR. This work was supported by NIDDK Grant DK061418 (C.C. Yun) and the American Heart Association Southeast Affiliate and Emory URC grant (I. Choi).

\section{References}

1. Orchard C.H. and Kentish J.C., Effects of changes of $\mathrm{pH}$ on the contractile function of cardiac muscle. Am. J. Physiol. 258: C967-C981, 1990.

2. Lagadic-Gossmann D., Buckler K.J. and Vaughan-Jones R.D., Role of bicarbonate in $\mathrm{pH}$ recovery from intracellular acidosis in the guinea-pig ventricular myocyte. J. Physiol. 458: 361-384, 1992.

3. Choi I., Aalkjaer C., Boulpaep E.L. and Boron W.F., An electroneutral sodium/bicarbonate cotransporter NBCn1 and associated sodium channel. Nature 405: 571-575, 2000.

4. Pushkin A., Abuladze N., Lee I., Newman D., Hwang J. and Kurtz I., Cloning, tissue distribution, genomic organization, and functional characterization of $\mathrm{NBC} 3$, a new member of the sodium bicarbonate cotransporter family. J. Biol. Chem. 274: 16569-16575, 1999.

5. Romero M.F., Fulton C.M. and Boron W.F., The SLC4 family of $\mathrm{HCO}_{3}^{-}$transporters. Pflügers Arch. 447: 495-509, 2004.

6. Odgaard E., Jakobsen J.K., Frische S., Praetorius J., Nielsen S., Aalkjaer C. and Leipziger J., Basolateral $\mathrm{Na}^{+}$-dependent $\mathrm{HCO}_{3}^{-}$transporter NBCn1-mediated $\mathrm{HCO}_{3}^{-}$influx in rat medullary thick ascending limb. J. Physiol 555: 205-218, 2004.

7. Cooper D.S., Saxena N.C., Yang H.S., Lee H.J., Moring A.G., Lee A. and Choi I., Molecular and functional characterization of the electroneutral $\mathrm{Na} / \mathrm{HCO}_{3}$ cotransporter NBCn1 in rat hippocampal neurons. J. Biol. Chem. 280: 17823-17830, 2005.

8. McMurtrie H.L., Cleary H.J., Alvarez B.V., Loiselle F.B., Sterling D., Morgan P.E., Johnson D.E. and Casey J.R., The bicarbonate transport metabolon. J. Enzyme Inhib. Med. Chem. 19: 231-236, 2004. 